\title{
Zero Temperature Phase Diagram of a $d$-Wave Superconductor with Anderson Impurities
}

\author{
L.S. BORKOWSKI \\ Quantum Physics Division, Faculty of Physics, A. Mickiewicz University \\ Umultowska 85, 61-614 Poznań, Poland
}

\begin{abstract}
We study the model of a $d$-wave superconductor interacting with finite concentration of the Anderson impurities at zero temperature. The interaction between impurity and conduction electrons is taken into account within the large- $N$ approximation. We discuss the obtained phase diagram and its dependence on the main energy scales.
\end{abstract}

PACS numbers: 74.81.--g, 74.25.Dw

\section{Introduction}

Magnetic and nonmagnetic impurities in correlated electron systems are an important probe of the properties of the host material. There is large and growing body of research on impurities in high-temperature superconductors $[1,2]$. The refinement of experimental techniques probing local properties stimulated the theoretical work on defects in those systems. The investigation of the effects of $\mathrm{Zn}, \mathrm{Ni}$ and other dopants in $\mathrm{YBa}_{2} \mathrm{Cu}_{3} \mathrm{O}_{7}(\mathrm{YBCO})$ and $\mathrm{Bi}_{2} \mathrm{Sr}_{2} \mathrm{CaCu}_{2} \mathrm{O}_{8+x}$ (BSCCO) answered some important questions and raised new ones.

Measurements on some compounds show that the superconducting order parameter is not uniform over the entire sample [3-7]. Scanning tunneling spectroscopy measurement showed that modulation of the structure of BSCCO is correlated locally with the magnitude of the energy gap [8]. The spatial variation of $\Delta_{0}(\boldsymbol{r})$ may result e.g. from the structural supermodulation affecting the strength of local pairing interaction [9].

The intrinsic spatial variation of the superconducting gap raises the possibility of observing impurity states on both sides of the impurity quantum phase transition in the same sample. Theoretical work on magnetic impurities in systems with reduced density of states near the Fermi surface [10-17] showed that the resonant impurity states may be viewed as a sensitive probe of the superconducting state. If the coupling to the magnetic impurity is small compared to the energy scale associated with the gap, the impurity is decoupled from conduction electrons. This impurity quantum phase transition occurs at finite coupling, provided the particle-hole symmetry is broken, and may be studied by scanning tunneling microscopy (STM) techniques. The low-energy behavior of the model depends on the exponent $r$ in the conduction electron density of states, $N(\epsilon) \sim|\epsilon|^{r}$, where the Fermi level is fixed at $\epsilon=0$.

\section{The model}

The interaction of impurities with the conduction electron band may be studied in the Anderson model with a BCS-type pairing interaction

$$
\begin{aligned}
H & =\sum_{k, m} \epsilon_{k} c_{k m}^{\dagger} c_{k m}+E_{0} \sum_{m} f_{m}^{\dagger} f_{m} \\
& +V \sum_{k, m}\left(c_{k, m}^{\dagger} f_{m} b+\text { h.c. }\right) \\
& +\sum_{k, m}\left[\Delta(k) c_{k m}^{\dagger} c_{-k-m}^{\dagger}+\text { h.c. }\right] .
\end{aligned}
$$

This model allows studying also the mixed valence regime where the impurity occupation number is smaller than 1 and charge fluctuations are dominant. We assume a two-dimensional $d$-wave order parameter of the form $\Delta(k)=\Delta_{0} \cos (2 \phi)$, where $\phi$ is the angle in the $k_{x}-k_{y}$ plane. The constraint term $\lambda\left(n_{f}+b^{\dagger} b-1\right)$ is added to the Hamiltonian to prevent doubly-occupied states, where $\lambda$ is the Lagrange multiplier and the limit $\lambda \rightarrow \infty$ is taken. Minimizing the free energy with respect to the resonant level energy $\epsilon_{f}$ and $z=\left\langle b^{\dagger}\right\rangle=\langle b\rangle$ and taking the mean field approximation we obtain

$$
\begin{aligned}
& \frac{1}{N}=-\operatorname{Im} \int_{-\infty}^{\infty} \mathrm{d} \omega f(\omega) \boldsymbol{G}_{f}\left(\omega+\mathrm{i} 0^{+}\right), \\
& \frac{E_{0}-\epsilon_{f}}{V^{2}} \\
& \left.\quad=\operatorname{Im} \int_{-\infty}^{\infty} \mathrm{d} \omega f(\omega) \boldsymbol{G}^{0}\left(\omega+\mathrm{i} 0^{+}\right) \boldsymbol{G}_{f}\left(\omega+\mathrm{i} 0^{+}\right)\right] .
\end{aligned}
$$

Equations (2) and (3) are solved self-consistently with the gap equation

$$
\Delta(k)=\int_{-\infty}^{\infty} \mathrm{d} \omega f(\omega) \sum_{k^{\prime}} V_{k k^{\prime}} \boldsymbol{G}\left(k^{\prime}, \omega\right) .
$$

The self-energy in the full conduction electron Green function $\boldsymbol{G}$ is averaged over impurity positions. We solve these equations self-consistently and obtain the phase diagram as a function of the parameters of the model. 


\section{Results and discussion}

The obtained phase diagram is shown in Fig. 1. The approximate slope of the impurity quantum phase transition line, -0.26 , agrees with results of the numerical renormalization group (NRG) method [14] and large- $N$ one-impurity calculation [17].

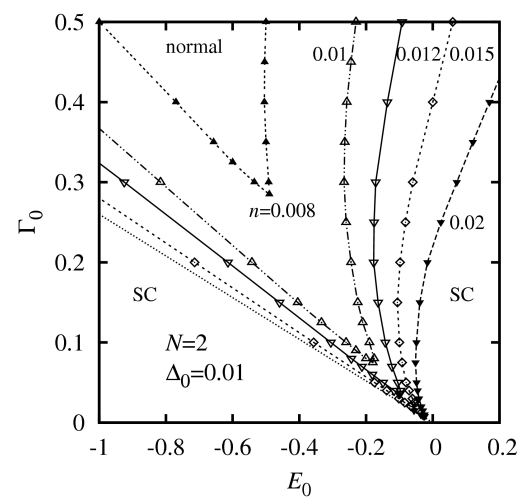

Fig. 1. The phase diagram of the $d$-wave superconductor with nondegerate Anderson impurities for several impurity concentrations. The lines are guide to the eye. The order parameter amplitude is $\Delta_{0}=0.01 D$, where $D$ is half of the conduction electron band width. All energies are scaled in units of $D$. The dotted line indicates the impurity quantum phase transition.

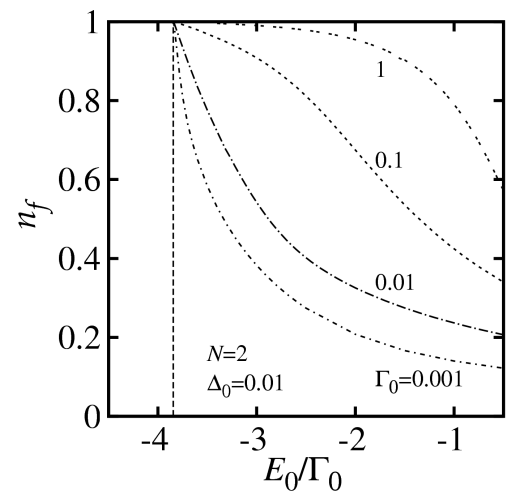

Fig. 2. The impurity occupation number as a function of the ratio $E_{0} / \Gamma_{0}$ for several values of $\Gamma_{0}$. At the impurity transition $n_{f} \rightarrow 1$.

When the bare impurity level $E_{0}$ lies closer to the Fermi energy, the self-consistent treatment of finite concentration of impurities leads to reentrant behavior [18]. In that part of the phase diagram the pair-breaking is weaker and is spread over wide energy range.

Figure 2 shows the impurity occupation number $n_{f}$ near the impurity transition. It reaches 1 at the transition. For $\Gamma_{0} \gg \Delta_{0}, n_{f}\left(E_{0}\right)$ slowly approaches 1 , $\left|\mathrm{d} n_{f} / \mathrm{d} E_{0}\right|_{\Gamma_{0}=\text { const }} \ll 1$, as $E_{0} / \Gamma_{0} \rightarrow\left(E_{0} / \Gamma_{0}\right)_{\text {critical }}$. However, for small $\Gamma_{0}$ the dependence of $n_{f}$ on $E_{0} / \Gamma_{0}$ becomes singular and the slope $\left|\mathrm{d} n_{f} / \mathrm{d} E_{0}\right|_{\Gamma_{0}=\text { const }} \gg 1$.

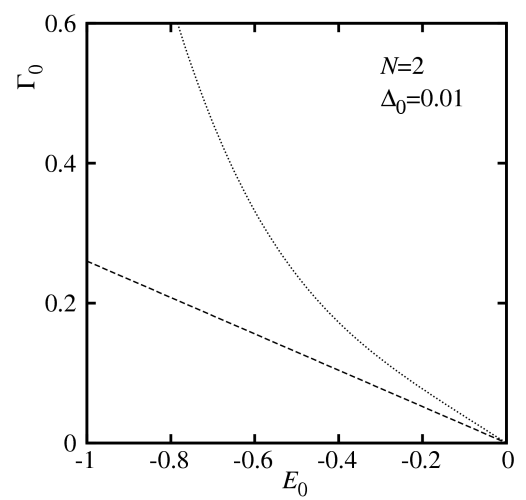

Fig. 3. The location of the cusp (dotted line) and the impurity transition from the local moment phase to the screened phase (broken line).

In the limit of vanishing interaction $\Gamma_{0} \rightarrow 0$, $E_{0}=0$ is the singular point of the model. For $E_{0}>0$ the superconducting state survives even for large impurity concentration.

For any finite $n$ there is a cusp on the phase diagram at some $\left(\Gamma_{0 c}, E_{0 c}\right)$. For $\Gamma_{0}$ slightly larger than $\Gamma_{0 c}$ there is a superconductor-normal state transition at $E_{01}<E_{0 c}$ and another normal-superconductor transition at $E_{02}$, where $E_{01}<E_{02}<E_{0 c}$. At fixed $n, E_{02}-E_{01} \simeq \alpha(n)\left(\Gamma_{0}-\Gamma_{0 c}\right)$, where $\alpha(n)$ weakly depends on $n$. The location of the cusp in the $E_{0}-\Gamma_{0}$ plane is shown in Fig. 3.

It would be interesting to test this theoretical picture in experiment. Near the impurity quantum phase transition the impurity state is very sensitive to small changes of hybridization $\Gamma_{0}$ or impurity level energy $E_{0}$. In compounds with spatially varying energy gap this could lead to impurities existing on the two sides of the transition line in different parts of the sample.

The phase diagram calculated in this work might also be relevant in some heavy-fermion compounds where similar competition occurs between energy scales associated with the Kondo screening and the superconducting correlations. Studies of $\mathrm{CeCu}_{2}\left(\mathrm{Si}_{1-x} \mathrm{Ge}_{x}\right)_{2}$ under varying hydrostatic pressure reveal two superconducting domes in the phase diagram $[19,20]$. The existing interpretation of this dependence on pressure relies on additional valence-fluctuation mediated pairing mechanism [21].

However, our work suggests that the second superconducting dome in the compound $\mathrm{CeCu}_{2}\left(\mathrm{Si}_{1-x} \mathrm{Ge}_{x}\right)_{2}$ at high pressure may follow from competition between pairing and the Kondo singlet formation. The change of pressure shifts the chemical potential and brings the system to the mixed-valence regime when the bare $f$-level $E_{0}$ of Ce ions approaches $E_{\mathrm{F}}$. The phase diagram in Fig. 1 shows that in this limit superconducting correlations are less affected.

The large- $N$ method used in the present calculation gives qualitatively similar results for larger $N$. The reentrant behavior as a function of $E_{0}$ for fixed $\Gamma_{0}$ results from the competition between the formation of the impurity 
resonance and superconducting correlations and depends mainly on the ratio of the relevant energy scales. Results for an $s$-wave order parameter will be presented in a separate publication.

Extension of the theory beyond the mean field should not change the phase diagram qualitatively. A more detailed description of physics in the vicinity of the impurity transition line requires careful treatment of low-energy scattering in specific superconducting compounds.

\section{References}

[1] H. Alloul, J. Bobroff, M. Gabay, P.J. Hirschfeld, Rev. Mod. Phys., to appear, and references therein.

[2] A.V. Balatsky, I. Vekhter, J.-X. Zhu, Rev. Mod. Phys. 78, 373 (2006), and references therein.

[3] T. Cren, D. Roditchev, W. Sacks, J. Klein, J.-B. Moussy, C. Deville-Cavellin, M. Lagues, Phys. Rev. Lett. 84, 147 (2000).

[4] S.-H. Pan, J.P. O'Neal, R.L. Badzey, C. Chamon, H. Ding, J.R. Engelbrecht, Z. Wang, H. Eisaki, S. Uchida, A.K. Guptak, K. Ng, E.W. Hudson, K.M. Lang, J.C. Davis, Nature 413, 282 (2001).

[5] C. Howald, P. Fournier, A. Kapitulnik, Phys. Rev. B 64, 100504(R) (2001).

[6] K.M. Lang, V. Madhavan, J.E. Hoffman, E.W. Hudson, H. Eisaki, S. Uchida, J.C. Davis, Nature 415, 412 (2002).

[7] K. McElroy, J. Lee, J.A. Slezak, D.-H. Lee, H. Eisaki, S. Uchida, J.C. Davis, Science 309, 1048 (2005).
[8] J.A. Slezak, Ph.D. Thesis, Cornell University, 2007.

[9] Y. He, S. Graser, P.J. Hirschfeld, H.-P. Cheng, Phys. Rev. B, to appear.

[10] D. Withoff, E. Fradkin, Phys. Rev. Lett. 64, 1835 (1990).

[11] L.S. Borkowski, P.J. Hirschfeld, Phys. Rev. B 46 , 9274 (1992)

[12] L.S. Borkowski, P.J. Hirschfeld, J. Low Temp. Phys. 96, 185 (1994).

[13] C. Gonzalex-Buxton, K. Ingersent, Phys. Rev. 54, R15614 (1996).

[14] C. Gonzalez-Buxton, K. Ingersent, Phys. Rev. B 57, 14254 (1998).

[15] J.-X. Zhu, C.S. Ting, Phys. Rev. B 63, 020506(R) (2000)

[16] A. Polkovnikov, S. Sachdev, M. Vojta, Phys. Rev. Lett. 86, 296 (2001).

[17] G.-M. Zhang, H. Hu, Lu Yu, Phys. Rev. Lett. 86, 704 (2001).

[18] L.S. Borkowski, Phys. Rev. B 78, 020507 (2008).

[19] H.Q. Yuan, F.M. Grosche, M. Deppe, C. Geibel, G. Sparn, F. Steglich, New J. Phys. 6, 132 (2004).

[20] P. Gegenwart, Q. Si, F. Steglich, Nature Phys. 4, 186 (2008)

[21] Y. Onishi, K. Miyake, J. Phys. Soc. Japan 69, 3955 (2000) 